\title{
ESTUDO DA VIABILIDADE E DESENVOLVIMENTO DE FILMES ATIVOS COM A UTILIZAÇÃO DE RESÍDUOS DA INDÚSTRIA VINÍCOLA
}

\section{STUDY OF THE VIABILITY AND DEVELOPMENT OF ACTIVE FILMS USING WINE INDUSTRY RESIDUES}

\author{
H. C. DIAS ${ }^{1}$ e A. B. REIS ${ }^{1, *}$ \\ ${ }^{1}$ Universidade Federal dos Vales do Jequitinhonha e Mucuri, Engenharia Química, Diamantina-MG, Brasil
}

*Autor correspondente. Universidade Federal dos Vales do Jequitinhonha e Mucuri, Departamento de Engenharia Química, Diamantina, Minas Gerais, Brasil, e-mail: arlete.reis@ict.ufvjm.edu.br(A.B.Reis)

\begin{tabular}{l} 
A R T I C L E I N F O \\
\hline Article history: \\
Received 2019-07-10 \\
Accepted 2019-12-20 \\
Available online 2019-12-20 \\
pa lavras-chave \\
Biopolímero \\
Quitosana \\
Casca de uva \\
Embalagem \\
ke ywords \\
Biopolymer \\
Chitosan \\
Grape skin \\
Package
\end{tabular}

\begin{abstract}
A B S T R A C T
In response to environmental awareness regarding the disposal of packaging and use of industrial waste, interest in developing active packaging with natural additives from tailings was increased. The extent of grape production in Brazil and the impact of the residual discard of fruit processing are well known. Considering the production of wines and knowing that grape skin, the main component of the residue, has in its composition agents with antioxidant activity, this work aims at the development of chitosan based films with grape skin for use in active packaging. In this work some analyzes were carried out for characterization, involving thickness, solubility, water vapor permeability and colorimetric analysis and SEM. The results demonstrate that the use of residues from the wine industry influenced the characteristics of the film, indicating its viability.
\end{abstract}

\section{R E S U M O}

Devido à conscientização frente ao descarte de embalagens e aproveitamento de resíduos industriais, aumentou-se o interesse no desenvolvimento de embalagens ativas com aditivos naturais oriundos de rejeitos. É notória a extensão da produção de uvas no Brasil e o impacto gerado pelo descarte residual do processamento da fruta. Tendo em vista a produção de vinhos e sabendo que a casca da uva, principal componente do resíduo, possui em sua composição agentes com atividade antioxidante, este trabalho visa o desenvolvimento de filmes a base de quitosana com casca de uva para utilização em embalagens ativas. Neste trabalho realizaram-se algumas análises para caracterização, envolvendo espessura, solubilidade, permeabilidade de vapor d'água, análise colorimétrica e MEV. Os resultados demonstram que a utilização de resíduos da indústria vinícola, influenciou nas características do filme, indicando sua viabilidade. 


\section{INTRODUÇÃO}

Em resposta a uma maior conscientização sobre os danos ambientais causados pelo descarte de embalagens, muitas empresas se concentram, atualmente, no desenvolvimento de embalagens à base de biopolímeros. Este tipo de material tem chamado atenção principalmente por sua característica de biodegradabilidade (Luchese et al., 2017). Outro indicativo desta concientização e maior responsabilidade ambiental, se torna evidente no reaproveitamento de resíduos nas mais diversas áreas de atuação industrial, visando sobretudo sua aplicação no favorecimento e geração de novos produtos ou, coprodutos, ambientalmente mais amigáveis.

Um exemplo de resíduo industrial com potencial para reaproveitamento é a biomassa residual do processo de vinificação, caracterizada por uma carga altamente versátil de polifenóis, muitos dos quais demonstraram exibir um amplo espectro de bioatividade, interessante para o uso em novos produtos. Tem-se desenvolvido inúmeras metodologias para a recuperação efetiva de polifenóis de resíduos sólidos da indústria vinícola, porém, em sua grande maioria não vão além da escala laboratorial, devido ao uso de solventes voláteis e caros, inadequados em termos de segurança e agravamento ambiental (Makris, 2018).

Segundo a EMBRAPA (2019), a produção de uvas no Brasil alcança 1,5 milhões de toneladas por ano. No ano de 2017, o estado de Minas Gerais produziu cerca de 13.685 toneladas de uvas. Do total de uvas produzidas no Brasil, aproximadamente $50 \%$ são destinadas a produção de vinhos, sucos e derivados. Nas indústrias vinícolas o principal resíduo gerado é o bagaço de uva, este é composto por $58 \%$ de cascas, $20 \%$ de engaços e $22 \%$ de sementes (Dantas et al., 2008). Apesar de ser um resíduo orgânico, ao ser descartado sem tratamento pode acarretar grandes impactos ambientais (Ferreira et al., 2012). Logo, o uso desses para fins mais nobres resultaria em benefícios tanto para a agroindústria vitivinícola como para os usuários dos coprodutos oriundos do bagaço de uva (Mello e Silva, 2014).

No âmbito apresentado, vê-se a oportunidade de utilização do bagaço de uva, material residual abundante, no desenvolvimento de embalagens que possuam vantagens tanto funcionais como ambientais, a partir da sua incorporação na matriz polimérica que dá origem a filmes para revestimento de embalagens celulósicas. A utilização de filmes biopoliméricos de quitosana tem despertado grande interesse frente a utilização de polímeros obtidos via síntese química, pois entre outras vantagens, apresenta potencial de estender o tempo de prateleira de produtos perecíveis, como é o caso dos alimentícios (Reis et al., 2005).

A quitosana é um biopolímero, caracterizado por sua não toxidade, biodegradabilidade, biocompatibilidade, além da sua capacidade antifúngica e antimicrobiana. Quimicamente a quitosana é obtida através da desacetilação, via hidrólise básica, da quitina, material presente principalmente no tecido que compõe o exoesqueleto de invertebrados e carapaça de crustáceos (Oliveira e Junior, 2016). Além de ser um polissacarídeo abundante, a quitosana possui propriedades que favorecem a formação de filmes. A característica hidrofílica deste biopolímero, resulta em revestimentos com eficiente barreira contra compostos de baixa polaridade, tais como os lipídios; por outo lado, não constituem boa barreira contra a umidade (Kester e Fennema, 1986). De acordo com Laranjeira e Fávere (2009), a hidrofilicidade deste biopolímero está associada à uma grande porcentagem de hidroxilas e radicais amino, presentes em sua estrutura molecular, apresentada na Figura 1. A utilização deste biopolímero em produção de filmes vem sendo estudada por décadas e é justificada por seu baixo custo de produção e versatilidade (Fráguas et al., 2015).

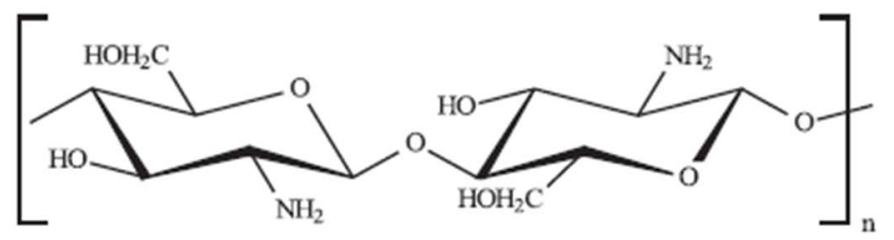

Figura 1 - Estrutura química do biopolímero de quitosana, onde n representa o grau de polimerização. (Assis e Silva, 2003)

Os filmes biopoliméricos a base de quitosana permitem a incorporação de agentes ativos em sua estrutura, abrindo inúmeras possibilidades de se agregar funcionalidade ao filme. Dentre as fontes para obtenção de aditivos com funcionalidade, temos as fontes naturais, provindo de matéria orgânica como bagaço de frutas e vegetais, e os aditivos sintéticos que vem tendo sua utilização restrita devido aos possíveis efeitos tóxicos que estes podem gerar a longo prazo. Aditivos naturais oriundos de rejeitos industriais, tornam seu uso ainda mais interessante, como é o caso da casca da uva. A uva está entre as maiores fontes de compostos fenólicos, que apresentam atividades funcionais, como os flavonóides que são encontrados na casca da fruta e possuem característica antioxidativas (Pesco et al., 2012), portanto podem ser utilizados substituindo antioxidantes quimicamente sintetizados, como por exemplo o ácido cítrico.

Segundo Karak (2019), os flavonóides apresentam duas bandas de absorção, sendo estas relacionadas aos anéis da estrutura, a primeira banda na faixa de $320 \mathrm{~nm}$ à $385 \mathrm{~nm}$ e a segunda banda entre $250 \mathrm{~nm}$ e $285 \mathrm{~nm}$, como é apresentado na Figura 2.

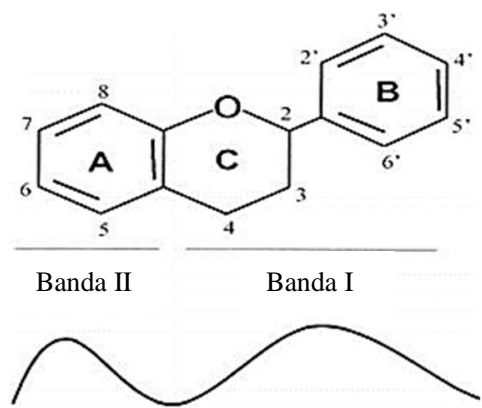

Figura 2 - Estrutura molecular geral dos flavonóides e bandas de absorção por espectroscopia. (Adaptado de Karak, 2019)

Sob a ótica da inovação tecnológica o emprego de agentes bioativos em embalagens representa uma abordagem inovadora, por meio das embalagens ativas, capazes de garantir a qualidade, integridade e segurança de produtos alimentícios 
(Yildirim et al., 2018). Segundo Gontard (1997), uma embalagem ativa é aquela capaz de proteger, interagir com o produto e, em alguns casos, responder às mudanças que ocorrem no interior da embalagem ou no ambiente ao qual está exposto. Além disso, permitem a liberação ou a absorção de substâncias dentro ou a partir dos alimentos embalados, ou ainda do meio ao redor da embalagem (Vanderroost et al., 2014). Um exemplo da funcionalidade de embalagens ativas são filmes comestíveis e sensíveis ao pH obtidos a partir do amido de araruta que continha farinha de resíduos de uva (Gutiérrez et al., 2018).

Neste sentido, temos como objetivo o desenvolvimento e caracterização preliminar de filmes a base de quitosana com adição de agentes antioxidantes, realizando a comparação entre o filme padrão de quitosana, filme com adição de ácido cítrico, e o filme produzido com casca de uva. Para caracterização inicial destes filmes é de suma importância a realização de testes de permeabilidade em vapor d'água, solubilidade, conhecimento de suas propriedades estruturais a partir da microscopia eletrônica de varredura e análise de cor, além da análise de atividade antioxidante dos compostos presentes na casca de uva.

\section{METODOLOGIA}

A seguir são apresentadas as metodologias adotadas neste estudo para o desenvolvimento dos filmes e análises realizadas com a finalidade de determinar a viabilidade da utilização de resíduos da indústria vinícola na produção de filmes para posterior aplicação em embalagens.

\subsection{Processamento da casca de Uva}

Utilizou-se a uva da espécie Vitis vinífera adquirida em comércio local para desenvolvimento dos filmes. Primeiramente as uvas foram lavadas, separou-se as cascas da polpa e dos caroços. Em seguida as cascas foram secas em estufa à temperatura de $60{ }^{\circ} \mathrm{C}$ durante 36 horas, e acompanhou-se a cinética de secagem por análise gravimétrica. Após secagem, as cascas foram mantidas em dessecador com sílica gel por 24 horas, posteriormente estas foram moídas em moinho de facas em 2 ciclos de 2 minutos, o material recolhido foi levado para peneiramento em modulo de tamisação. A granulometria mais fina obtida foi correspondente ao mesh 48, o material com granulometria superior foi moído novamente em 1 ciclo de 2 minutos, e em seguida peneirados.

\subsection{Produção dos filmes}

Os produção dos filmes seguiu a técnica de casting, que consiste na solubilização da quitosana em solução ácida, seguida da evaporação do solvente por secagem em placas de petri. Foram produzidos filmes de $1 \%(\mathrm{~m} / \mathrm{v})$ e $2 \%(\mathrm{~m} / \mathrm{v})$, onde respectivamente, $1 \mathrm{~g}$ e $2 \mathrm{~g}$ de quitosana em pó (Polymar), foram solubilizados em solução de $1 \mathrm{~mL}$ de ácido acético (Synth) para $100 \mathrm{~mL}$ de água deionizada, em agitador magnético por 1 hora. A solução formada foi disposta em placas de petri, cada uma contendo $20 \mathrm{~mL}$. As placas de petri foram submetidas a secagem em estufa a $40{ }^{\circ} \mathrm{C}$ por 24 horas. Após a secagem os filmes obtidos foram retirados da placa de petri. Estes filmes foram usados como padrão para as análises realizadas.

Além destas, outras quatro formulações foram produzidas, sendo duas com ácido cítrico (Synth) e duas com a casca de uva previamente processada, a estes foi adicionado glicerol (Synth) como agente plastificante. Na Tabela 1, são apresentadas as nomenclaturas de cada formulação e sua respectiva composição.

Para produção dos filmes com a casca de uvas, realizouse a solubilização de $1 \mathrm{~g}$ de casca em $100 \mathrm{~mL}$ de água deionizada em Banho metabólico Dubnofff orbital em agitação constante por 24 horas. Após filtragem, o filme era então produzido seguindo a metodologia do filme padrão a partir do sobrenadante.

\subsection{Análise visual}

A partir da obtenção dos filmes, foram observadas características perceptíveis ao olho humano das amostras. Avaliou-se coloração, fragilidade, e homogeneidade dos filmes. Os filmes que ao serem retirados das placas apresentassem rupturas, não homogeneidades, ou aspecto quebradiço eram descartados.

\subsection{Espessura}

A espessura dos filmes foi medida aleatoriamente em 5 pontos distintos da área do filme, utilizando o micrômetro Electronic Outside Micrometer - Series 3103 0-25mm/0-1", da marca INSIZE. Analisou-se a espessura pela média e pelo desvio padrão dos pontos medidos.

\subsection{Permeabilidade ao vapor d'água (PVA)}

Para permeabilidade ao vapor d'água seguiu-se o método gravimétrico de acordo com a norma ASTM E96-95 (ASTM, 1995), onde o filme foi mantido em dessecador contendo sílica gel por 48 horas, após ser retirado do dessecador o filme foi utilizado para lacrar um recipiente contendo $20 \mathrm{~g}$ de sílica gel, devidamente vedado com auxílio de fita veda rosca, de forma que não ocorresse entrada de umidade, a não ser por permeabilidade do filme.

Os sistemas, foram mantidos em dessecador contendo solução de $\mathrm{NaCl}$ (Synth) com umidade relativa controlada de $75 \%$ e temperatura ambiente. A variação mássica do sistema foi acompanhada durante 5 dias, e o cálculo da permeabilidade foi realizado como mostra a Equação 1.

$$
P V A=\frac{\Delta m * x}{A * t * \Delta P}
$$

Onde: $\Delta m$ é a variação de massa do sistema; $x$ é a espessura do filme; $A$ é a área do filme em contato com o meio; $t$ é o tempo de exposição; e $\Delta P$ é a variação de pressão.

\subsection{Solubilidade}

A determinação da solubilidade das amostras foi realizada de acordo com Amaral et al. (2017), onde uma porção de $1 \mathrm{~g}$ de filmes, cortado em pedaços com área igual a $2 \mathrm{~cm}^{2}(1$ $\mathrm{cm} \times 2 \mathrm{~cm}$ ), foi armazenada em dessecador com sílica gel por 7 dias, em seguida inserida em recipiente contendo $80 \mathrm{~mL}$ de água em temperatura ambiente e submetidas a agitação de $200 \mathrm{rpm}$ em manta agitadora por 1 hora. Após agitação a amostra de filme foi seca em estufa a $60{ }^{\circ} \mathrm{C}$, pelo período de 24 horas. A partir das análises do experimento realizado em triplicata, obteve-se a solubilidade das amostras pela Equação 2.

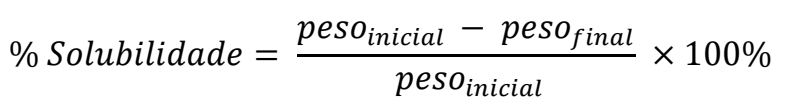

\subsection{Análise Colorimétrica}

A coloração dos filmes foi obtida em Colorímetro da marca Konica Minolta, do laboratório de matérias primas alimentares, do Instituto de Ciência e Tecnologia da 
Universidade Federal dos Vales do Jequitinhonha e Mucuri, por meio dos parâmetros: $\mathrm{L}^{*}$, variando de 0 (preto) a 100 (branco); $a^{*}$, variando do verde (-) ao vermelho $(+)$; e b*, com variação do azul (-) ao amarelo (+), aplicando-se o iluminante D65 à luz do dia. Os biofilmes foram aplicados na superfície de uma placa branca padrão.

\subsection{Microscopia eletrônica de varredura (MEV)}

A análise de microscopia eletrônica de varredura, para analisar a estrutura dos filmes, foi realizada no Laboratório Multiusuário de Microscopia Avançada (LMMA) do Departamento de Química na Universidade Federal dos Vales do Jequitinhonha e Mucuri. Foram realizadas análises em Microscópio Eletrônico de Varredura TESCAN VEGA-LMH de vista plana, vista lateral e espectroscopia por energia dispersiva (EDS) das amostras.

\subsection{Análise em espectrofotômetro UV/VIS}

Para análise de absorbância foi utilizado o espectrofotômetro UV/VIS modelo Cary 60, Agilent. Foi realizada a extração da casca de uva em água na concentração de $10 \mathrm{~g} / \mathrm{L}$, durante 30 minutos em agitação constante, em seguida o extrato foi filtrado. Primeiramente utilizou-se a solução do extrato na proporção de $10 \%$ para obter o pico de absorção das amostras. As medidas da absorção foram tomadas para concentrações de $0 \%, 10 \%, 40 \%, 70 \%$ e $100 \%$ de extrato em água, a fim de determinar a curva que relaciona a concentração da amostra com a respectiva absorbância, a curva foi obtida utilizando o software Excel.

\subsection{Análise estatística}

Os resultados dos testes realizados são apresentados como média \pm desvio padrão. Foram realizadas análises de variância (ANOVA) e o teste de Tukey para identificar diferenças significativas entre as médias, usando o software $R$ 3.6.0. Aplicando-se nível de confiança de $95 \%$.

Tabela 1- Composição dos filmes e nomenclatura correspondente.

$\begin{array}{lllllll}\text { Nomenclatura } & \text { Q1 } & \text { Q2 } & \text { Q1Ac } & \text { Q2Ac } & \text { Q1Cas } & \text { Q2Cas } \\ \text { Água / mL } & 100,00 & 100,00 & 100,00 & 100,00 & 100,00 & 100,00 \\ \text { Quitosana / g } & 1,00 & 2,00 & 1,00 & 2,00 & 1,00 & 2,00 \\ \text { Ác. acético / mL } & 1,00 & 1,00 & 1,00 & 1,00 & 1,00 & 1,00 \\ \text { Ác. cítrico / g } & - & - & 1,00 & 1,00 & - & - \\ \text { Glicerol / g } & - & - & 0,50 & 1,00 & 0,50 & 0,75 \\ \text { Casca / g } & - & - & - & - & 1,00 & 1,00 \\ \end{array}$

\section{RESULTADOS}

Os resultados obtidos no presente estudo para as análises realizadas nas amostras de filmes Q1, Q2, Q1Ac, Q2Ac, Q1Cas e Q2Cas, estão disponíveis a seguir.

\subsection{Análise Visual}

Os filmes Q1, Q2, Q1Ac, Q2Ac, Q1Cas e Q2Cas, produzidos pelo método de Casting são apresentadas a seguir na Figura 3, as fotografias foram tiradas logo após a retirada dos filmes das placas de petri, após etapa de secagem.
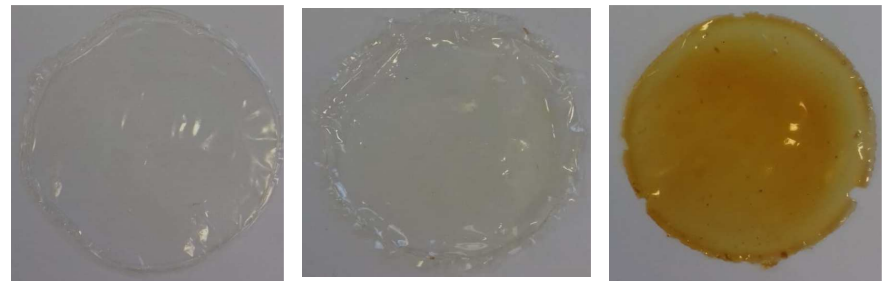

a) Q1

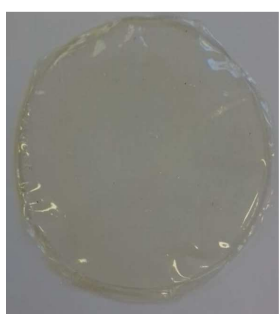

b) Q1Ac

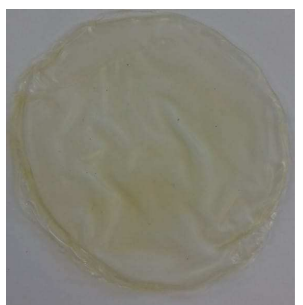

c) Q1Cas

d) Q2

e) Q2Ac

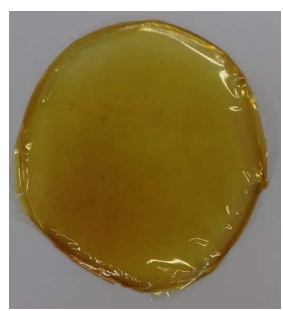

f) Q2Cas

Figura 3- Amostra de filmes produzidos - a) Q1, b) Q1Ac, c) Q1Cas, d) Q2, e) Q2Ac, f) Q2Cas.
Por inspeção visual, os filmes apresentaram-se íntegros e homogêneos. Em relação a coloração os filmes Q1 e Q1Ac apresentaram maior transparência, o filme Q2 apesar de apresentar certa transparência possui uma coloração amarelada muito suave, o filme Q2Ac tem característica opaca, enquanto os filmes Q1Cas e Q2cas apresentaram coloração amarelo intenso. O filme Q1Ac demonstrou característica mais elástica e superfície aderente.

\subsection{Espessura}

Os filmes a base de quitosana apresentaram, segundo análise, os valores de espessura média como mostra Tabela 2. A incorporação de ácido cítrico e casca de uva, na solução filmogenica, resultou em um aumento da espessura dos filmes em relação ao filme padrão. Para as amostras Q1Cas e Q2Cas o teste estatístico de Tukey, indicou que não há diferença significativa entre as espessuras das amostras associada ao aumento de concentração de quitosana.

Tabela 2- Espessura média das amostras de filmes.

$\begin{array}{cl}\text { Amostra } & \text { Espessura média / mm } \\ \text { Q1 } & 0,072 \pm 0,0045^{\mathrm{d}} \\ \text { Q2 } & 0,103 \pm 0,0047^{\mathrm{c}} \\ \text { Q1Ac } & 0,122 \pm 0,0010^{\mathrm{b}} \\ \text { Q2Ac } & 0,147 \pm 0,0033^{\mathrm{a}} \\ \text { Q1Cas } & 0,120 \pm 0,0047^{\mathrm{b}} \\ \text { Q2Cas } & 0,126 \pm 0,0021^{\mathrm{b}}\end{array}$

Nota: Resultados que apresentam letras diferentes implicam diferença significativa $(p<0,05)$ entre as amostras, pelo teste estatístico de Tukey. 


\subsection{Permeabilidade ao vapor d'água (PVA)}

A análise de PVA foi realizada em quadruplicata para as amostras de filme Q1, Q2, Q1Ac, Q2Ac, Q1Cas e Q2Cas, uma área do filme foi $0,0056745 \mathrm{~m}^{2}$ e a variação de pressão de $2,3 \mathrm{kPa}$ ocasionada pela diferença entre as umidades relativas dos meios interno e externo, obteve-se os resultados de PVA apresentados na Tabela 3. Foi possível observar que a permeabilidade é consideravelmente maior nos filmes produzidos com casca de uva, para o filme Q1Cas, por exemplo, observa-se um aumento igual a 110,90\% em relação ao Q1.

Tabela 3 - Permeabilidade Vapor d'Água.

$\begin{array}{cl}\text { Amostra } & \text { PVA } / \mathrm{g} \cdot \mathrm{mm} \cdot \mathrm{m}^{-2} \cdot \mathrm{h}^{-1} \cdot \mathrm{kPa}^{-1} \\ \text { Q1 } & 0,2568 \pm 0,0108^{\mathrm{d}} \\ \text { Q2 } & 0,3155 \pm 0,0137^{\mathrm{c}} \\ \text { Q1Ac } & 0,3345 \pm 0,0191^{\mathrm{c}} \\ \text { Q2Ac } & 0,4650 \pm 0,0204^{\mathrm{b}} \\ \text { Q1Cas } & 0,5416 \pm 0,0202^{\mathrm{a}} \\ \text { Q2Cas } & 0,5139 \pm 0,0087^{\mathrm{a}}\end{array}$

Nota: Resultados que apresentam letras diferentes implicam diferença significativa $(p<0,05)$ entre as amostras, pelo teste estatístico de Tukey.

Bertan (2008) explica a relação entre a adição de agentes plastificantes com o aumento da permeabilidade ao vapor d'água, devido a sua característica hidrofílica. Contudo, comparando-se os filmes Q1Ac e Q1Cas podemos inferir que a casca de uva influenciou no aumento da permeabilidade, visto que ambos foram produzidos com a mesma quantidade de agente plastificante, 0,5 g. Para embalagens de alimentos é interessante que a permeabilidade ao vapor d'água seja a menor possível.

\subsection{Solubilidade}

Pode-se observar que as amostras possuem diferentes percentuais de solubilidade em água. Os filmes Q1Cas e Q2Cas, possuem característica de solubilidade significativamente maior, como mostra a Tabela 4.

Tabela 4 - Solubilidade em água.

\begin{tabular}{cl}
\hline Amostra & Solubilidade / \% \\
\hline Q1 & $33,00 \pm 7,55^{\mathrm{b}, \mathrm{c}}$ \\
Q2 & $18,33 \pm 5,51^{\mathrm{d}}$ \\
Q1Ac & $23,77 \pm 2,24^{\mathrm{c}, \mathrm{d}}$ \\
Q2Ac & $34,60 \pm 3,24^{\mathrm{b}, \mathrm{c}}$ \\
Q1Cas & $48,27 \pm 0,73^{\mathrm{a}}$ \\
Q2Cas & $42,50 \pm 4,09^{\mathrm{a}, \mathrm{b}}$
\end{tabular}

Nota: Resultados que apresentam letras diferentes implicam diferença significativa $(\mathrm{p}<0,05)$ entre as amostras, pelo teste estatístico de Tukey.

\subsection{Análise colorimétrica}

Segundo Nascimento et al., (2013), a importância da cor dos filmes aplicados em embalagens, está diretamente associada à aceitabilidade do consumidor.

Os parâmetros da análise colorimétrica foram obtidos em triplicata e são apresentados na Tabela 5. Percebe-se que em todos os parâmetros ocorreu variação significativa entre os filmes, principalmente nos parâmetros $\mathrm{a}^{*} \mathrm{e} \mathrm{b}^{*}$ onde a alternância de sinal de (-) para (+) nos filmes produzidos a partir da casca de uva indicam uma predominância da coloração amarelo associado ao parâmetro $b^{*}$, assim como da coloração vermelho indicada pela coordenada $\mathrm{a}^{*}$, por este motivo define-se a coloração do filme desenvolvido como amarelo- castanho.

Tabela 5 - Parâmetros da análise colorimétrica.

\begin{tabular}{|c|c|c|c|}
\hline Amostra & $\mathrm{L}^{*}$ & $a^{*}$ & $b^{*}$ \\
\hline Q1 & $28,27 \pm 0,01^{\mathrm{c}}$ & $-0,43 \pm 0,04^{\mathrm{c}}$ & $-0,73 \pm 0,11^{d}$ \\
\hline Q2 & $22,97 \pm 0,12^{\mathrm{e}}$ & $-0,84 \pm 0,03^{d}$ & $-0,11 \pm 0,01^{\mathrm{c}}$ \\
\hline Q1Ac & $31,54 \pm 0,54^{b}$ & $-0,49 \pm 0,01^{c}$ & $-0,17 \pm 0,09^{c}$ \\
\hline Q2Ac & $32,87 \pm 0,06^{\mathrm{a}}$ & $-0,87 \pm 0,02^{d}$ & $1,96 \pm 0,02^{b}$ \\
\hline Q1Cas & $20,91 \pm 0,19^{f}$ & $0,21 \pm 0,03^{b}$ & $2,96 \pm 0,12^{a}$ \\
\hline Q2Cas & $23,66 \pm 0,09^{d}$ & $2,35 \pm 0,01^{\mathrm{a}}$ & $2,78 \pm 0,04^{\mathrm{a}}$ \\
\hline
\end{tabular}

Nota: Resultados que apresentam letras diferentes, na mesma coluna, implicam diferença significativa $(\mathrm{p}<0,05)$ entre as amostras, pelo teste estatístico de Tukey.

\subsection{Análise em espectrofotômetro UV/VIS}

A avaliação em espectrofotômetro na amostra da extração da casca de uva em água apresentou pico de absorção no comprimento de onda de $267 \mathrm{~nm}$. A Figura 4 apresenta, a curva que relaciona a concentração de extrativos por solução com a absorbância. $\mathrm{O}$ ajuste linear da curva originou a Equação 3 mostrada a seguir que descreve o comportamento as amostras.

$$
\text { Abs }=0,3612 * \text { Conc }+0,0562
$$

Onde: Abs, é a absorbância da amostra; Conc, é a concentração de cascas em $\mathrm{g} / \mathrm{L}$ no extrativo.

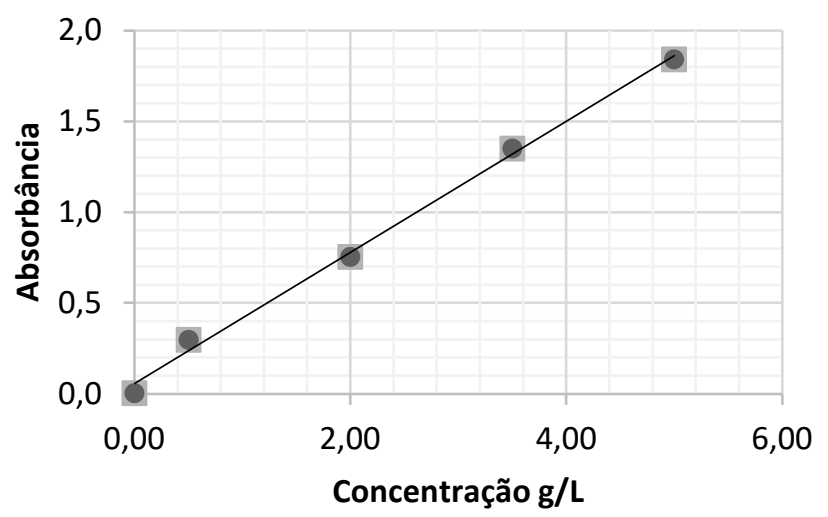

Figura 4 - Curva de absorbância da extração de casca de uva em água.

A equação de ajuste apresentou coeficiente de correlação $\mathrm{R}^{2}=0,9962$, demonstrando que a equação ajustada descreve de forma satisfatória o comportamento de absorbância observado em análise, por este motivo podemos indicar que absorbância das amostras esta de fato associada a compostos presentes na casca de uva, pois esta apresentou um comportamento linear em relação a concentração de cascas.

\subsection{Microscopia Eletrônica de Varredura - MEV}

Foram realizadas micrografias das amostras de filmes produzidos, nas amplitudes de 200x, 500x e 1000x, em todas amplitudes os filmes apresentaram a mesma estrutura. As 
micrografias de vista plana, com ampliação de 1000x são Q1Cas e Q2Cas.

ilustradas na Figura 5, para as amostras Q1, Q2, Q1Ac, Q2Ac,

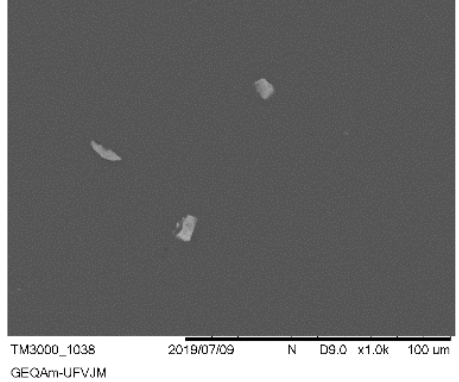

a) Q1 (1000x)

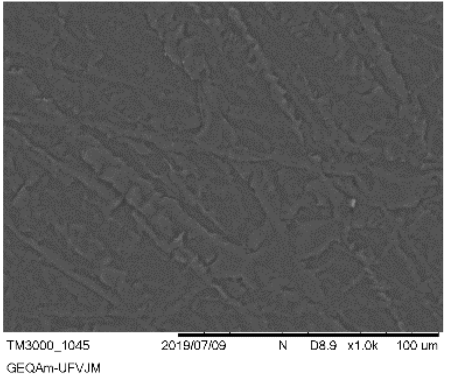

d) Q2 (1000x)

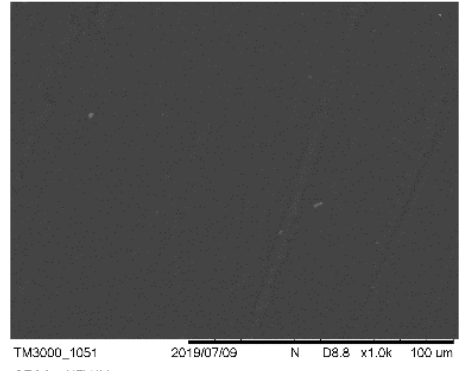

b) Q1Ac (1000x)

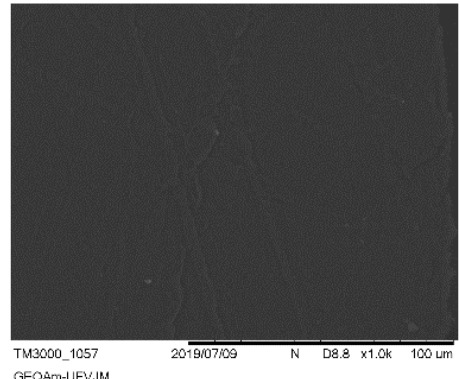

e) Q2Ac $(1000 x)$

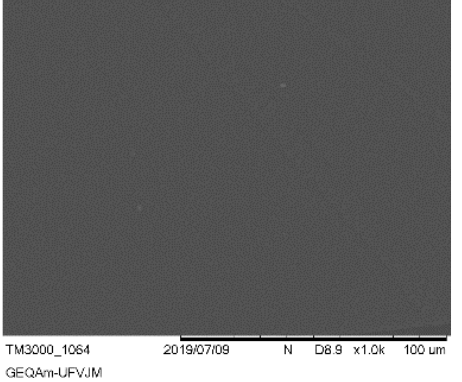

c) Q1Cas (1000x)

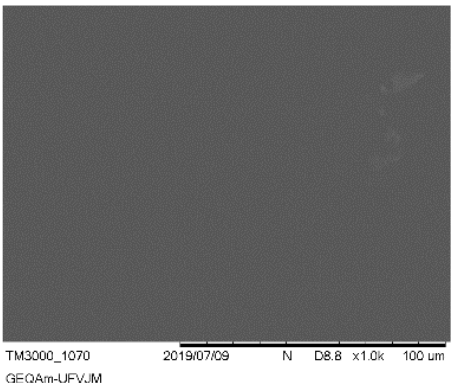

f) Q2Cas $(1000 x)$

Figura 5 - Micrografias de vista plana com amplitude de 1000x. a) Q1, b) Q1Ac, c) Q1Cas, d) Q2, e) Q2Ac, f) Q2Cas.

É possível observar a homogeneidade estrutural dos filmes. O que implica afirmar que houve boa solubilização da quitosana, e ainda que a interação entre este biopolímero e os aditivos empregados, ácido cítrico e flavonóides, se deu de forma homogênea na superfície do filme.

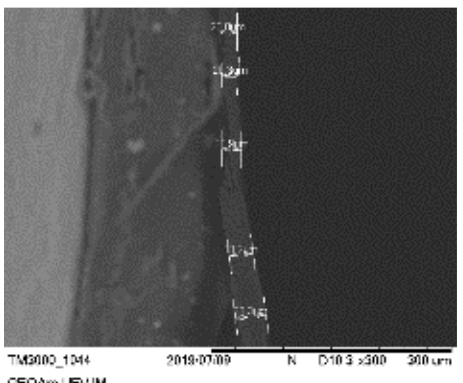

a) Q1 (300x)

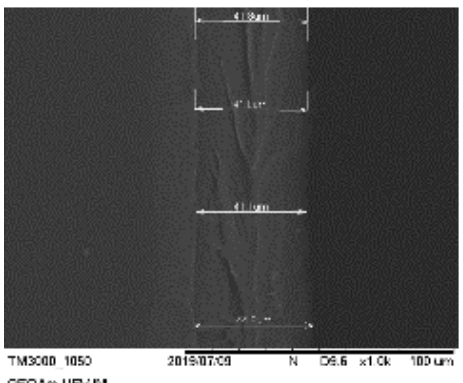

d) Q2 (1000x)

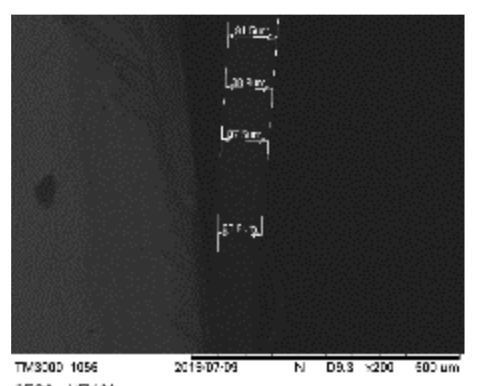

b) Q1Ac (200x)

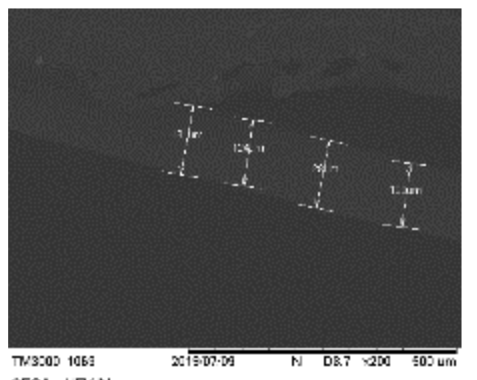

e) Q2Ac (200x)
Por meio das micrografias de vista lateral mostradas na Figura 6, pode-se avaliar a homogeneidade da espessura dos filmes produzidos. Foi possível confirmar que as amostras Q1, Q1Ac, Q1Cas, Q2, Q2Ac e Q2Cas, apresentaram uma espessura regular e homogênea.

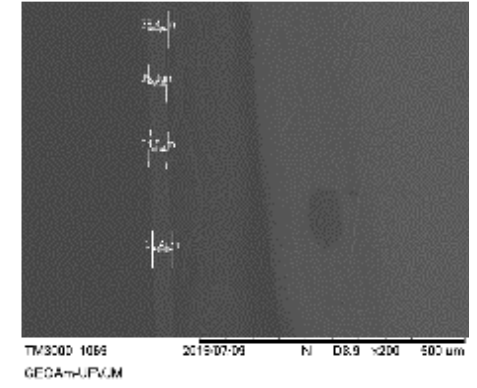

c) Q1Cas (200x)

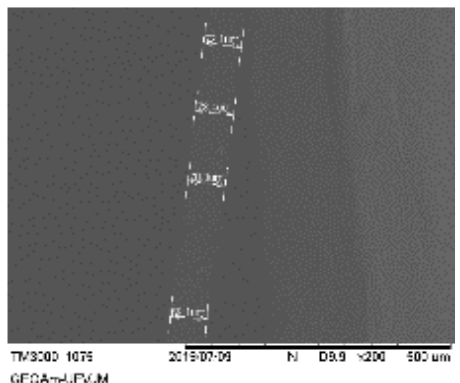

f) Q2Cas (200x)

Figura 5 - Micrografias de vista lateral das amostras. a) Q1, b) Q1Ac, c) Q1Cas, d) Q2, e) Q2Ac, f) Q2Cas.

Foram realizadas análises de EDS juntamente com o MEV, a fim de avaliar a composição dos filmes, os resultados apontaram a presença de carbono, nitrogênio e oxigênio nas amostras Q1, Q1Ac, Q1Cas, Q2, Q2Ac e Q2Cas, em diferentes 
concentrações, como indica Tabela 6.

Tabela 6 - Quantificação da composição química dos filmes em relação percentual ao peso molecular.

\begin{tabular}{cccc} 
Amostra & $\mathrm{C} / \%$ & $\mathrm{O} / \%$ & $\mathrm{~N} / \%$ \\
\hline Q1 & 37,201 & 41,654 & 21,145 \\
Q2 & 36,159 & 39,742 & 24,099 \\
Q1Ac & 33,642 & 48,397 & 17,961 \\
Q2Ac & 34,769 & 48,883 & 16,348 \\
Q1Cas & 38,322 & 43,600 & 18,078 \\
Q2Cas & 37,053 & 43,811 & 19,139
\end{tabular}

A predominância de carbono, oxigênio e nitrogênio era esperada devido às estruturas o biopolímero, do ácido cítrico e do flavonoide. Não foi observada a presença de hidrogênio devido a limitações do equipamento utilizado. Podemos observar que não houve grande variação de composição entre as amostras, o que fortalece a característica de homogeneidade dos filmes observada nas análises de MEV.

\section{DISCUSSÃO}

Pelo aspecto visual os filmes, apresentaram boas características de homogeneidade e integridade. A coloração do filme interfere diretamente na aceitação do produto pelo consumidor, os filmes de casca de uva possuem coloração amarela intenso atrativa, além disso pode interferir na incidência de luminosidade no produto.

A adição tanto do ácido cítrico como da casca de uva alterou de forma significativa a espessura do filme de quitosana. Essa característica do filme pode variar dependendo das características do produto, além disso foi possível observar que a espessura foi maior nos filmes com maior concentração de quitosana, devido ao aumento de massa seca dos filmes. De acordo com Jiménez et al. (2012) é esperado uma espessura mais homogênea a fim de evitar problemas mecânicos que possam comprometer a segurança do produto. De forma geral as amostras testadas apresentaram baixos valores de desvio padrão. A espessura mais fina foi a do filme Q1 com média de $0,072 \mathrm{~mm}$, e a espessura mais alta foi de $0,147 \mathrm{~mm}$ no filme Q2Ac. Para os filmes contendo $2 \mathrm{~g}$ de quitosana a adição da casca de uva, Q2Cas, apresentou uma espessura significativamente menor frente a adição de ácido cítrico, Q2Ac. O aumento da concentração de quitosana nos filmes produzidos com a casca de uva não ocasionou diferença estatística na espessura do filme.

Conforme ilustra a Tabela 3 , podemos inferir que a permeabilidade ao vapor d'água do filme aumenta em razão do aumento da concentração de quitosana, para os filmes padrão de quitosana e para com filmes com adição de ácido cítrico, explicado pelo caráter hidrofílico deste biopolímero. Contudo dada a interação com a casca de uva o valor médio de PVA não sofre mudança significativa para ambas as concentrações. $\mathrm{O}$ maior valor de PVA encontrado foi nos filmes com casca de uva, esta característica pode estar relacionada com a adição do agente plastificante como também ao tipo de interação, ligações de hidrogênio, entre a molécula de quitosana e a molécula do composto fenólicos, flavonóides presentes na casca da uva, pois este tipo de ligação aumenta o caráter hidrofílico do filme. A absorção de vapor de água na matriz polimérica faz com que ocorra uma maior taxa de permeação pois a água atua como um agente plastificante, aumentando a porcentagem de espaços vazios na estrutura do filme (Kim; Ustunol, 2001).

Com a adição dos compostos da casca de uva apresentou boa característica de solubilidade, a maior solubilidade observada foi de 48,27\% no filme Q1Cas, também foi o teste de solubilidade que apresentou o menor desvio padrão. Com relação a solubilidade para diferentes concentrações de quitosana pela análise estatística foi possível encontrar diferenças significativas.

Houve alteração estatisticamente significativas nos parâmetros de cor dos filmes. A análise de cor evidencia a presença de coloração amarela dos filmes com casca de uva a partir do parâmetro $b^{*}$.

Pela análise em espectrofotômetro o pico de absorção encontrado está dentro da faixa apontada pela literatura, referente a segunda banda de absorção da molécula, demonstrando a presença da estrutura de flavonóides no extrato de uva em água pelos quais foram obtidos os filmes Q1Cas e Q2Cas.

As micrografias obtidas pelo $\mathrm{MEV}$, confirmam o caráter homogêneo das amostras observadas na análise visual, tanto em relação à superfície do filme quanto a espessura destes.

\section{CONCLUSÃO}

Com o estudo realizado foi possível desenvolver uma metodologia para obtenção do filme a base do biopolímero quitosana, com utilização de resíduos da indústria vinícola. Os resultados obtidos confirmam que a adição das cascas influência significativamente nas propriedades do filme, com potencial de aplicação em embalagens. Análises feitas nas amostras mostram que os filmes possuem espessura homogênea, boa característica de solubilidade, baixa resistência a permeabilidade ao vapor d'água, a análise colorimétrica indica que o filme possui coloração amarelo-castanho pode apresentar atividade de cor. Além disso, a análise de absorbância indicou que o processo de extração da casca em uva foi viável. A viabilidade de aplicação está relacionada diretamente às características e necessidades de armazenamento do produto a ser embalado. Contudo, como se trata de um resíduo industrial e um biopolímero biodegradável, tendo em vista o baixo custo associado à produção do filme, pode-se inferir que existe viabilidade na aplicação de resíduos da indústria vinícola em materiais de embalagem.

\section{A G R A D E C I M E N T O S}

Os autores agradecem ao apoio do LMMA patrocinado pela FAPEMIG (CEX-112-10), SECTES/MG e RQ-MG (FAPEMIG: CEX-RED-00010-14), ao GEPAEQ Grupo de Estudos e Pesquisas Aplicada à Engenharia Química-UFVJM, e ao Laboratório de Matérias Primas Alimentares- UFVJM.

\section{REFERENCIAS}

AMARAL, I. B. C.; ARRUDAS, S. R.; DE MEIRA, J. R.; REIS, A. B. Análise do processo difusivo de filmes de quitosana contendo óleo de palmeiras (Aracaceae) do cerrado brasileiro. Revista Unimontes Científica, Montes Claros, MG, jul./dez. 2017.

AMERICAN SOCIETY FOR TESTING AND MATERIALS- 
ASTM Book of Standards, Philadelphia, PA. ASTM E96-00 - Standard test methods for determining gas permeability characteristics of plastic film and sheeting, 1995.

ASSIS, O. B. G.; SILVA, V. L. Caracterização estrutural e da capacidade de absorção de água em filmes finos de quitosana processados em diversas concentrações. Polímeros: Ciência e Tecnologia, v. 13, p. 223-228, 2003.

BERTAN, L. C. Desenvolvimento e caracterização de biofilmes ativos à base de polímeros de fontes renováveis e sua aplicação no acondicionamento de pães de forma. Tese (Doutorado em Tecnologia de Alimentos) - Unicamp, Campinas, SP, 2008.

DANTAS, F.R.; ARAÚJO, G.G.L. de; SILVA, D.S. da; PEREIRA, L.G.R.; GONZAGA NETO, S.; TOSTO, M. da S.L. Composição química e características fermentativas de silagens de maniçoba (Manihot sp.) com porcentuais de coproduto de vitivinícolas desidratado. Revista Brasileira de Saúde e Produção Animal, v.9, p. 247-257, 2008.

EMBRAPA. A Viticultura no Brasil. Disponível em: $<$ https://www.embrapa.br/cim-inteligencia-e-mercadouva-e-vinho/a-viticultura-no-brasil >. Acessado em 15 de junho 2019.

FERREIRA, L. F. D.; PIROZI, M. R.; RAMOS, A. M.; PEREIRA, J. A. M. Modelagem matemática da secagem em camada delgada de bagaço de uva fermentado. Pesquisa Agropecuária Brasileira, 47(6), p. 855-862. 2012.

FRÁGUAS, R. M.; SIMÃO, A. A.; FARIA, P. V.; QUEIROZ, E. DE R.; OLIVEIRA JUNIOR, Ê. N. DE; ABREU, C. M. P. DE. Preparo e caracterização de filmes comestíveis de quitosana. Polímeros. 2015.

GONTARD, N. Active packaging. In: SOBRAL, P.J.A.; CHUZEL, G., eds. Workshop sobre biopolímeros. Pirassununga, FZEA. p. 23-27. 1997.

GUTIERREZ, T. J.; HERNIOU-JULIEN, C.; ALVAREZ, K., \& ALVAREZ, V. A. Structural properties and in vitro digestibility of edible and $\mathrm{pH}$-sensitive films made from guinea arrowroot starch and wastes from wine manufacture. Carbohydrate Polymers, v. 184. 2018.

JIMÉNEZ, A.; FABRA, M. J.; TALENS, P.; CHIRALT, A. Edible and Biodegradable Starch Films: A Review. Food Bioprocess Technology. v.4, p.0-0, 2012.

KARAK, P. Biological activities of flavonoids: an overview. International Journal of Pharmaceutical Sciences and research. v. 10, p. 1567-1574. 2019.

KESTER, J.J.; FENNEMA, O.R. Edible films and coatings: a review. Food Technology, v. 40, n. 12, p. 47-59, 1986.

KIM, S-J.; USTUNOL, Z. Solubility and moisture sorption isotherms of wheyprotein- based edible films as influenced by lipid and plasticizer incorporation. Journal of Agricultural and Food Chemistry, Washington, v. 49, n.9, p. 4388-4391, 2001.

LARANJEIRA, M. C. M.; FÁVERE, V. T. Quitosana: Biopolímero funcional com potencial industrial biomédico. Química Nova, Florianópolis, SC, v. 32, p. 672-678, 2009.

LUCHESE, C. L.; SPEROTTO N.; SPADA, J. C.; TESSARO, I. C. Effect of blueberry agroindustrial waste addition to corn starch-based films for the production of a phindicator film. International Journal of Biological Macromolecules. 2017.

MAKRIS, D. P. Green extraction processes for the efficient recovery of bioactive polyphenols from wine industry solid wastes - Recent progress. 2018. Current Opinion in Green and Sustainable Chemistry. v. 13, p. 5055. October, 2018.

MELLO, L. M. R. de; SILVA, G. A. da. Disponibilidade e Características de Resíduos Provenientes da Agroindústria de Processamento de Uva do Rio Grande do Sul. Bento Gonçalves: Embrapa Uva e Vinho. 2014.

NASCIMENTO, S. D.; OLIVEIRA, T. A.; SANTOS, F. K. G.; AROUCHA, E. M. M.; LEITE, R. H. L. Efeito da adição de argila nas propriedades de biofilme de quitosana. Revista Verde de Agroecologia e desenvolvimento Sustentável. Agosto, 2013.

OLIVEIRA, Camila. S.; JUNIOR, Enio. N. Oliveira. Produção e caracterização de filmes de quitosana utilizando diferentes ácidos orgânicos. In: XXI Congresso brasileiro de engenharia química, 2016, Fortaleza, CE. Anais [...]. 2016.

PESCO, D. C. S.; APARECIDO, D.; GIACOMINI, T.; APARÍCIO, D.; CORTEZ, G.; ELAINE, L.; CORTEZ, R. Avaliação da atividade antioxidante de amostras de uvas (Vitis Vinifera 1.). In: VI Mostra interna de trabalhos de iniciação científica, 2012, Maringá. Anais eletrônicos [...].2012.

REIS, A. B.; YOSHIDA, C. M. P.; FRANCO, T. T. Filmes bioativos à partir do polímero quitosana: sistemas de embalagem de papelão. In: $8^{\circ}$ Congresso brasileiro de polímeros, 2005, Águas de Lindóia, SP. Anais [...]. 2005

VANDERROOST, M.; RAGAERT, P; DEVLIEGHERE, F.; MEULENAER, B. Intelligent packaging: The next generation. Trends in Food Science \& Technology, v.39, p. 47-62, 2014.

YILDIRIM, S.; RÖCKER, B.; PETTERSEN, M. K.; NILSENNYGAARD, J.; AYHAN, Z.; RUTKAITE, R. Active Packaging Applications for Food. Comprehensive Reviews in Food Science and Food Safety, p. 165-199. 2018. 